Supporting Information for

\title{
Enhancing Open Modification Searches via a Combined Approach Facilitated by Ursgal
}

Stefan Schulze ${ }^{l \dagger^{* *}}$, Aime Bienfait Igiraneza ${ }^{l \dagger}$, Manuel Kösters ${ }^{2}$, Johannes Leufken ${ }^{2}$, Sebastian A. Leidel ${ }^{2}$, Benjamin A. Garcia ${ }^{3}$, Christian Fufezan ${ }^{4}$, and Mechthild Pohlschröder ${ }^{{ }^{*}}$

${ }^{1}$ Department of Biology, University of Pennsylvania, Philadelphia, PA 19104, USA

${ }^{2}$ Department of Chemistry and Biochemistry, University of Bern, 3012 Bern, Switzerland

${ }^{3}$ Department of Biochemistry and Biophysics, Perelman School of Medicine, University of Pennsylvania, Philadelphia, PA 19104, USA

${ }^{4}$ Institute of Pharmacy and Molecular Biotechnology, Heidelberg University, 69120 Heidelberg, Germany

$\dagger$ These authors contributed equally to this work.

${ }^{*}$ Correspondence should be addressed to: sschulze@sas.upenn.edu and pohlschr@sas.upenn.edu

\section{Table of contents}

Figure S1: Effects of sanitizing results from OMS engines.

Figure S2: Overlap between different OMS engines for results from prokaryotic proteome datasets.

Figure S3: Increase of commonly identified spectra through the combination of results from different OMS engines.

Figure S4: PSM differences between OMS engines for results from prokaryotic proteome datasets.

Figure S5: Mass difference profiles for $H$. volcanii.

Figure S6: Mass difference profiles for E. coli.

Figure S7: Comparisons between OMS and CS results.

Figure S8: Identification of $O$-glycopeptides through the OMS approach. 
a

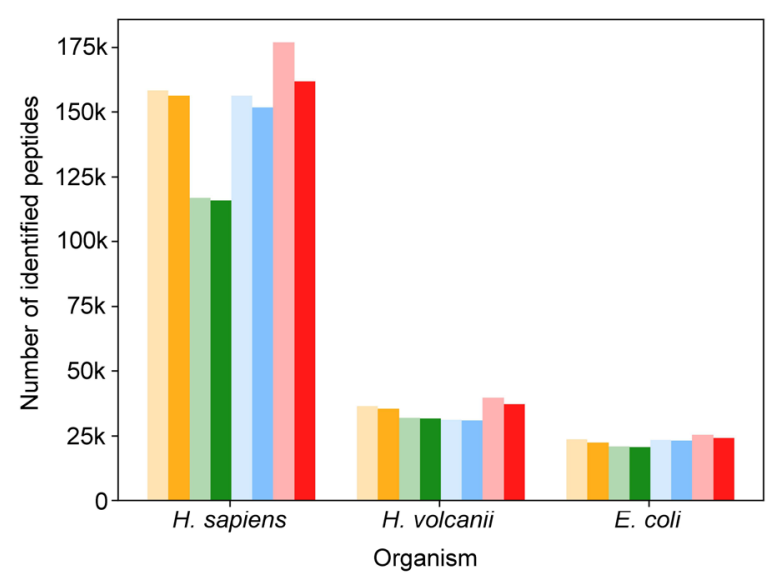

b

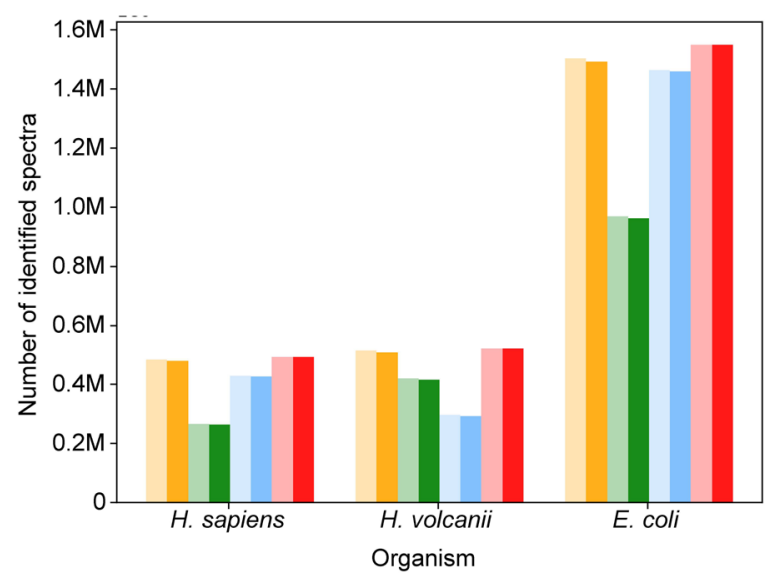

$$
\begin{array}{ll}
\text { MSFragger } & \text { MODa } \\
\text { MSFragger sanitized } & \text { MODa sanitized } \\
\text { PIPI } & \text { All (combined PEP } \leq 1 \% \text { ) } \\
\text { PIPI sanitized } & \text { All (combined PEP } \leq 1 \% \text { ) sanitized }
\end{array}
$$

Figure S1. Sanitizing affected results from all OMS engines similarly, indicating similar quality of PSMs. Across three studied datasets, the number of identified peptide sequences (a) and spectra (b) based on PSMs filtered by $1 \%$ combined PEP without sanitizing (light colors) is compared to after sanitizing (dark colors), i.e. only accepting the best scoring PSM for each spectrum. Results are presented for each individual engine (orange for MSFragger; blue for MODa; green for PIPI) as well as the merged results of all engines (red). 

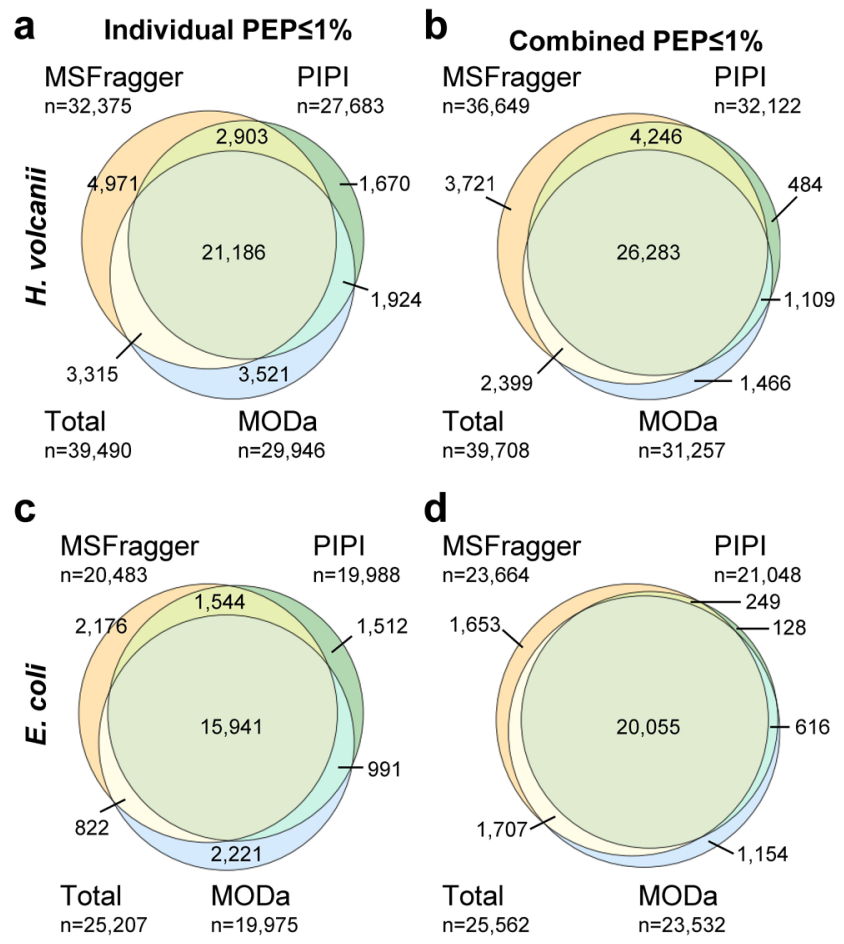

Figure S2. OMS results from different engines showed large overlaps for prokaryotic proteomes. Venn diagrams comparing peptide sequences identified by the OMS engines MSFragger (orange), MODa (blue) and PIPI (green) are shown, considering only PSMs filtered by 1\% PEP for each engine separately (a, c) and representing results from a combined PEP approach with PSMs filtered by $1 \%$ combined PEP (b, d). Results for the H. volcanii dataset are presented in (a) and (b), while E. coli dataset results are shown in (c) and (d). 


\section{a Individual PEP $\leq 1 \%$ b Combined PEP $\leq 1 \%$}

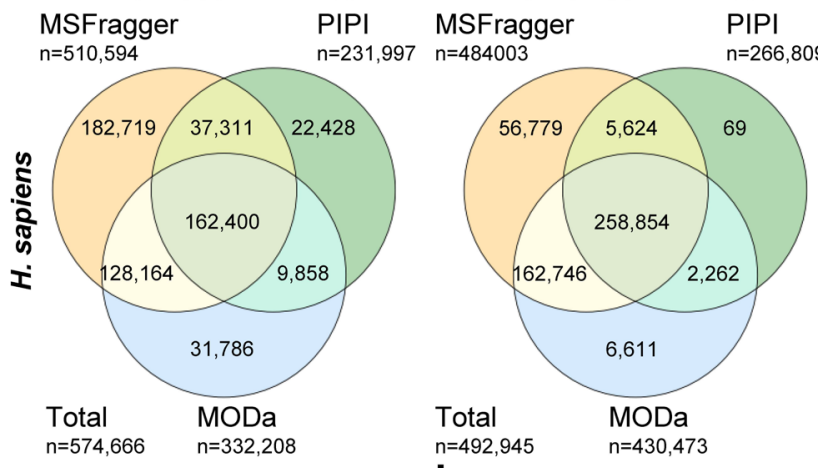

MSFragger PIPI

C

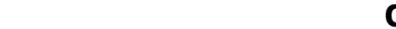

d

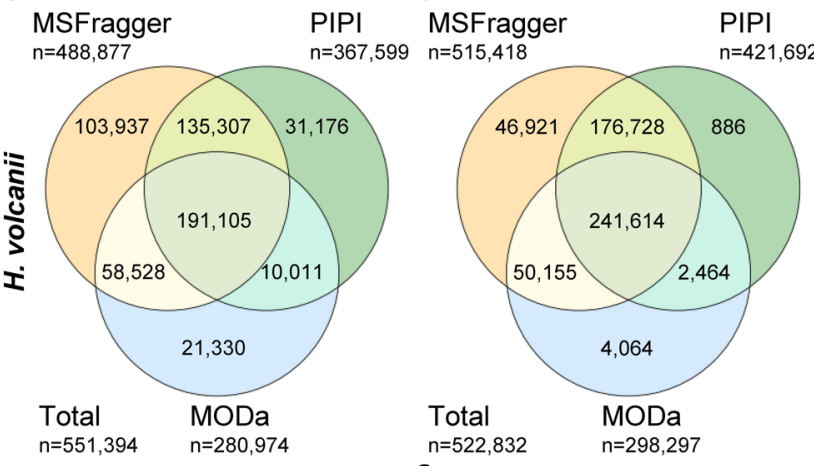

e

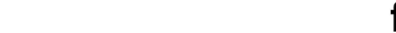

$f$
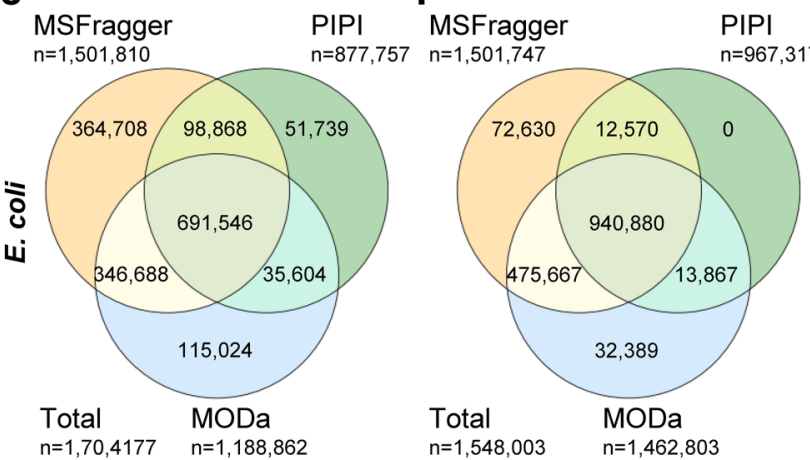

g

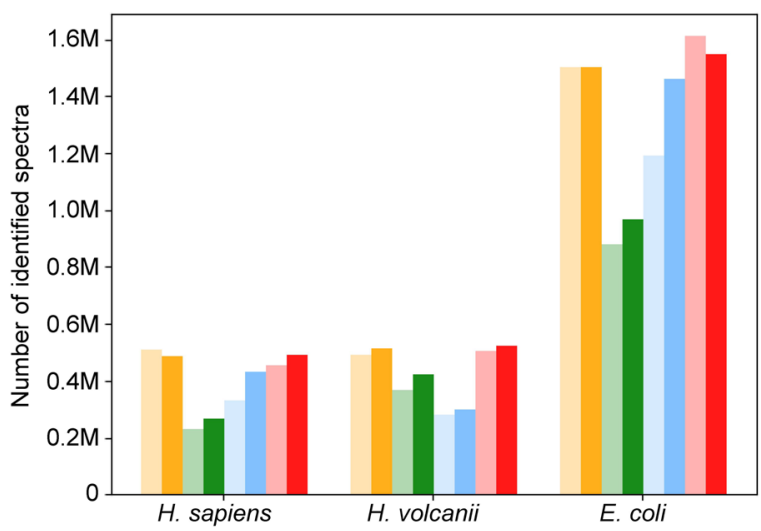

MSFragger (individual PEP $\leq 1 \%$ ) MODa (individual $P E P \leq 1 \%$ )

MSFragger (combined PEP $\leq 1 \%$ ) MODa (combined PEP $\leq 1 \%$ )

PIPI (individual PEP $\leq 1 \%$ ) All (individual PEP $\leq 1 \%$ )

PIPI (combined PEP $\leq 1 \%$ ) All (combined PEP $\leq 1 \%$ ) 
Figure S3. The combination of results from different OMS engines increased the number of commonly identified spectra. Venn diagrams comparing spectra leading to peptide identifications by the OMS engines MSFragger (orange), MODa (blue) and PIPI (green) are shown, considering only PSMs filtered by $1 \%$ PEP for each engine separately (a, c, e), and representing results from a combined PEP approach with PSMs filtered by $1 \%$ combined PEP $(\mathbf{b}, \mathbf{d}, \mathbf{f})$. Results are shown for datasets from $H$. sapiens $(\mathbf{a}, \mathbf{b})$, E. coli $(\mathbf{c}, \mathbf{d})$ and $H$. volcanii $(\mathbf{e}, \mathbf{f})$. (g), Across the three studied datasets, the number of identified spectra based on PSMs filtered by 1\% PEP for each engine (light colors) is compared to those found in PSMs filtered by $1 \%$ combined PEP (dark colors). Results are presented for each individual engine as well as the merged results of all engines (red). It should be noted that for merged results of PSMs filtered by $1 \%$ individual engine PEP, the overall PEP may be as high as 3\%. Results were sanitized, accepting only the best scoring PSM per spectrum. For (a - f), this sanitizing step was performed at the single engine level, to represent peptide sequences identified by each individual engine. For merged results of all engines within (g) however, sanitizing was performed over results from all engines, accepting only the best scoring PSM per spectrum for conflicting matches between different engines. 


\section{a}

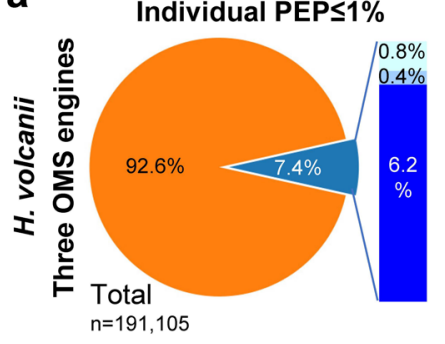

C

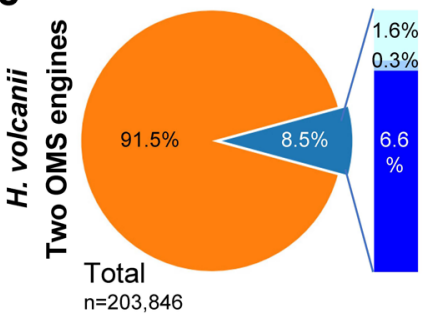

e

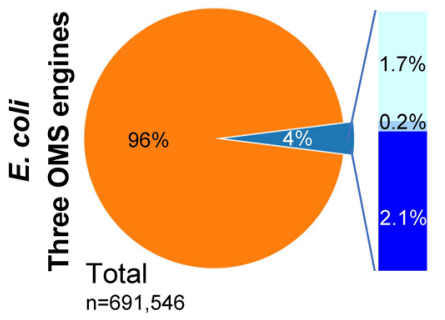

g

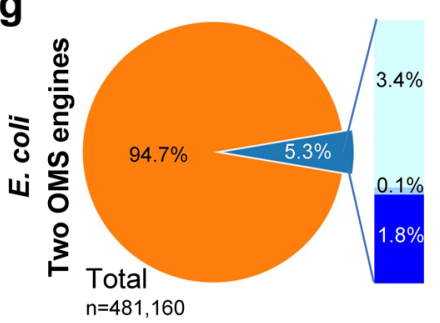

PSM agreements
PSM differences $\quad\left\{\begin{array}{l}\text { Peptide length difference } \\ \leq 3 \text { AA difference } \\ \text { Other differences }\end{array}\right.$ b

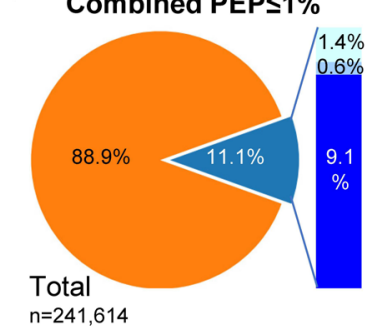

d

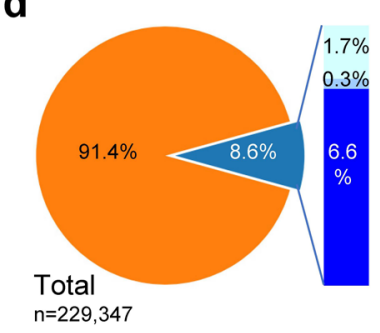

f

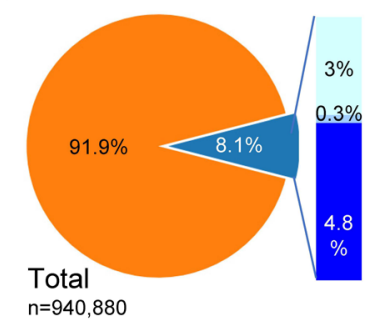

$\mathbf{h}$

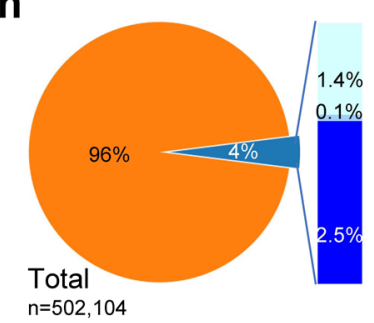

Figure S4. Mainly driven by differences in peptide length, PSM disagreements between different OMS engines were smaller for prokaryotic proteomes than for the human proteome. Spectra for which PSMs were identified by all three engines $(\mathbf{a}, \mathbf{b}, \mathbf{e}, \mathbf{f})$ as well as spectra for which PSMs were identified by two engines $(\mathbf{c}, \mathbf{d}, \mathbf{g}, \mathbf{h})$ were analyzed for agreements (orange) and disagreements (blue), i.e. whether at least one engine identified a different peptide sequence than the other(s). PSMs were filtered by $1 \%$ individual engine PEP in (a), (c), (e) and (g) in contrast to a filtering by $1 \%$ combined PEP in (b), (d), (f), and (h). Spectra with PSM disagreements were sorted into three categories: (i) peptide sequences with differing lengths, for which the shorter peptide is a substrings of the longer one (dark blue); (ii) peptides with one to three differing amino acids (medium blue); and (iii) peptides differing in any other way (light blue). The results represent the $H$. volcanii (a-d) and E. coli (e-h) datasets. 

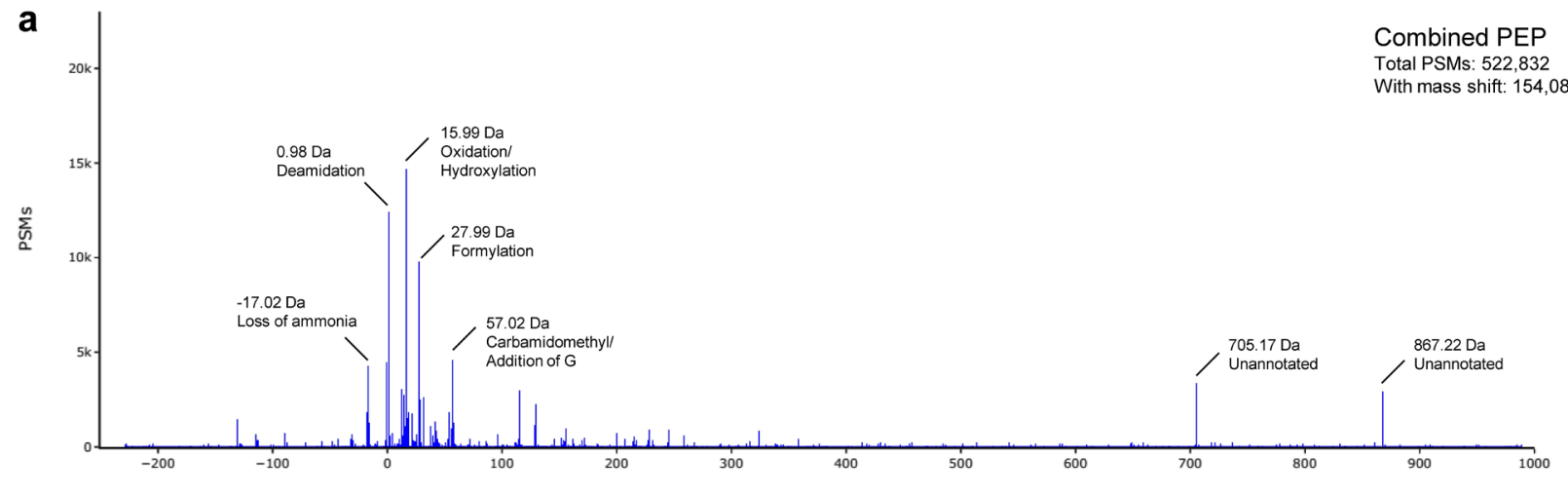

b

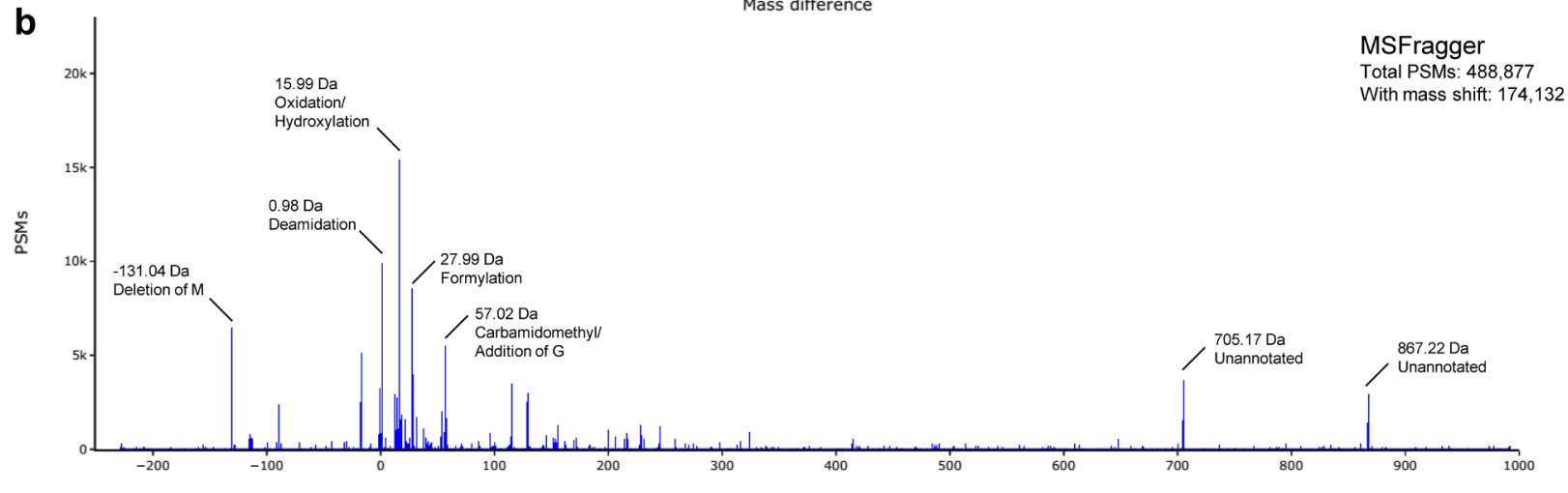

Mass difference

Combined PEP

Total PSMs: 522,832
With mass shift: 154,084

C

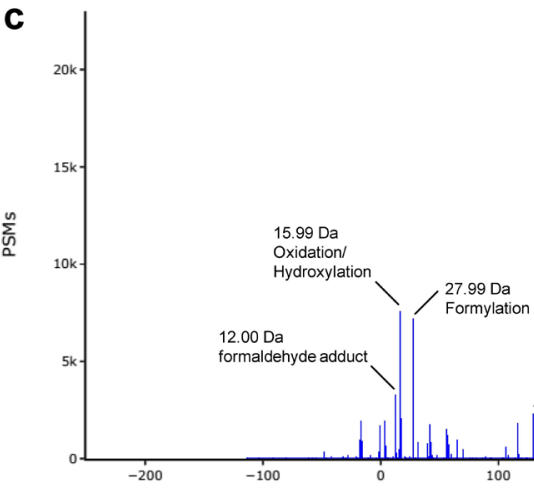

Mass difference

MODa

Total PSMs: 280,974

With mass shift: 65,396

d
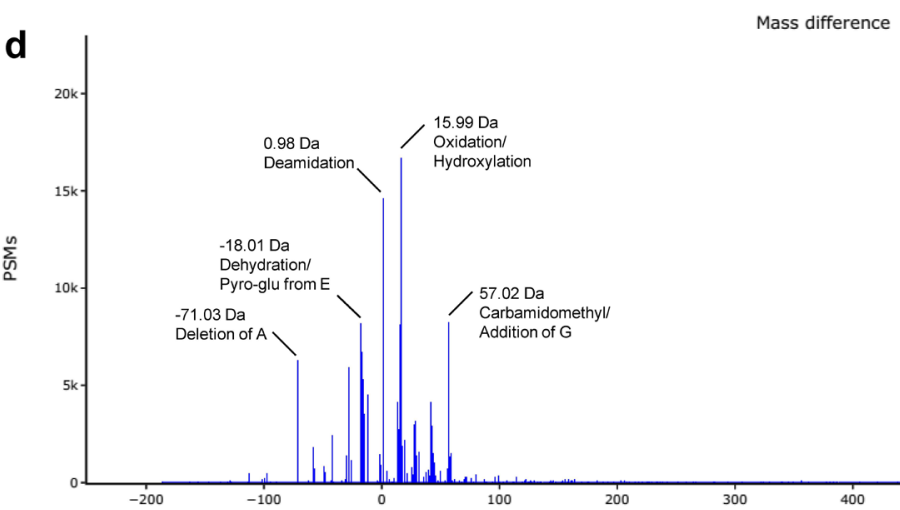

Mass difference

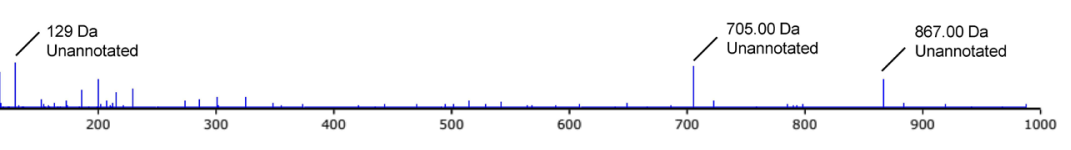

PIPI

Total PSMs: 367,599

With mass shift: 158,799

Mass difference

Figure S5. Mass difference profiles after unified post-processing of OMS results for $\boldsymbol{H}$. volcanii. Mass differences from combined results of all employed OMS engines (a), filtered by $1 \%$ combined PEP, as well as mass differences from results of MSFragger (b), MODa (c), and PIPI (d), filtered by $1 \%$ individual PEP, 
were post-processed using PTM-Shepherd. Results are presented as histograms giving the number of PSMs for each $0.05 \mathrm{Da}$ mass difference bin, omitting PSMs with no mass difference, or mass differences corresponding to isotopic peak selection. Results were sanitized on the individual engine level (b-c) or for the combination of all engines (a) before post-processing with PTM-Shepherd. Annotations for the most prominent mass differences are given. Notably, prominent mass differences at 705.17 Da and 867.22 Da correspond to tetra- and pentasaccharide $N$-glycans, respectively. The number of all identified PSMs ("Total PSMs") is given for all subfigures as well as the number of PSMs that contain mass shifts shown in the profiles ("With mass shift"). It should be noted that PSMs containing multiple mass differences are counted multiple times. 

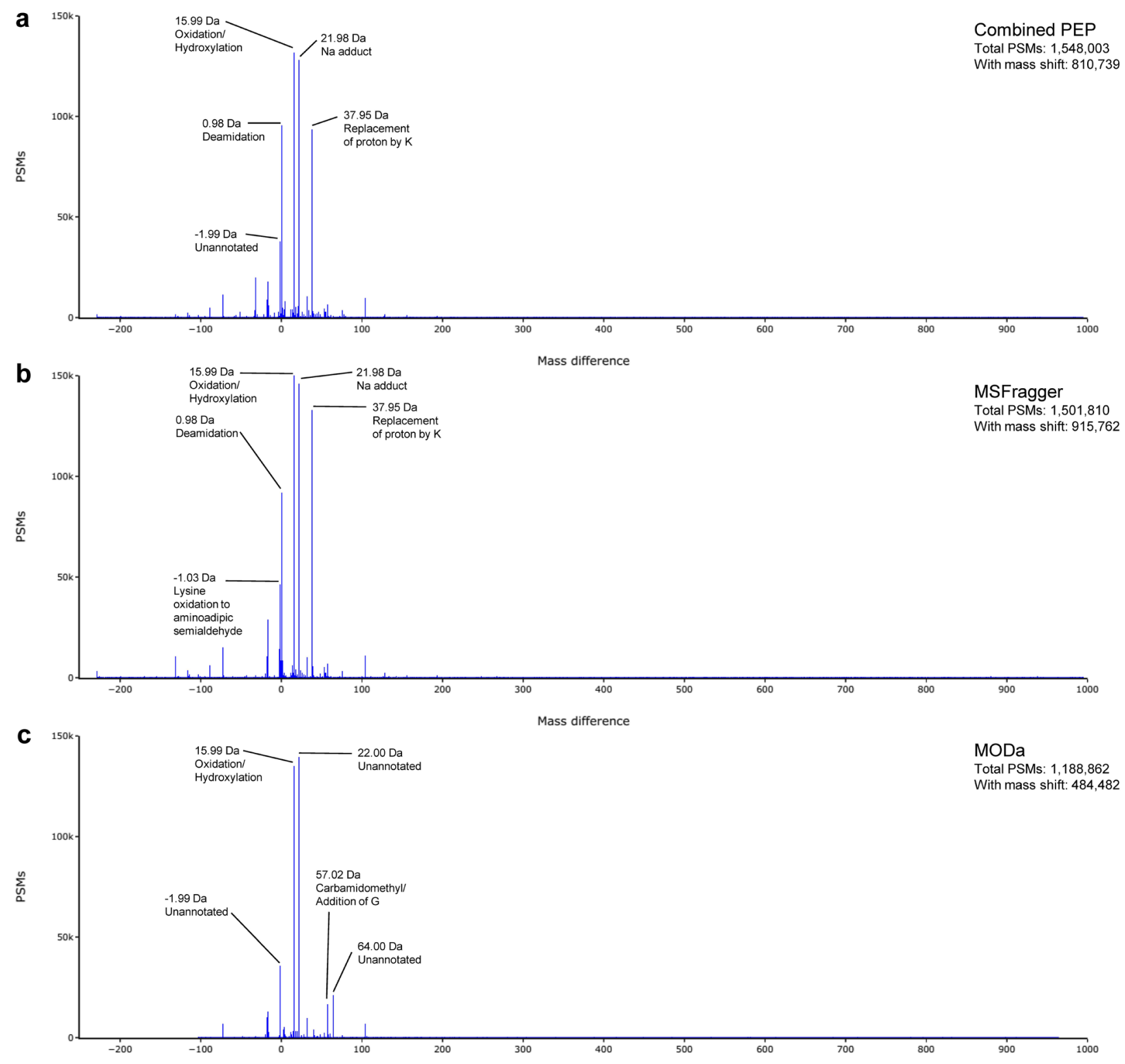

Mass difference

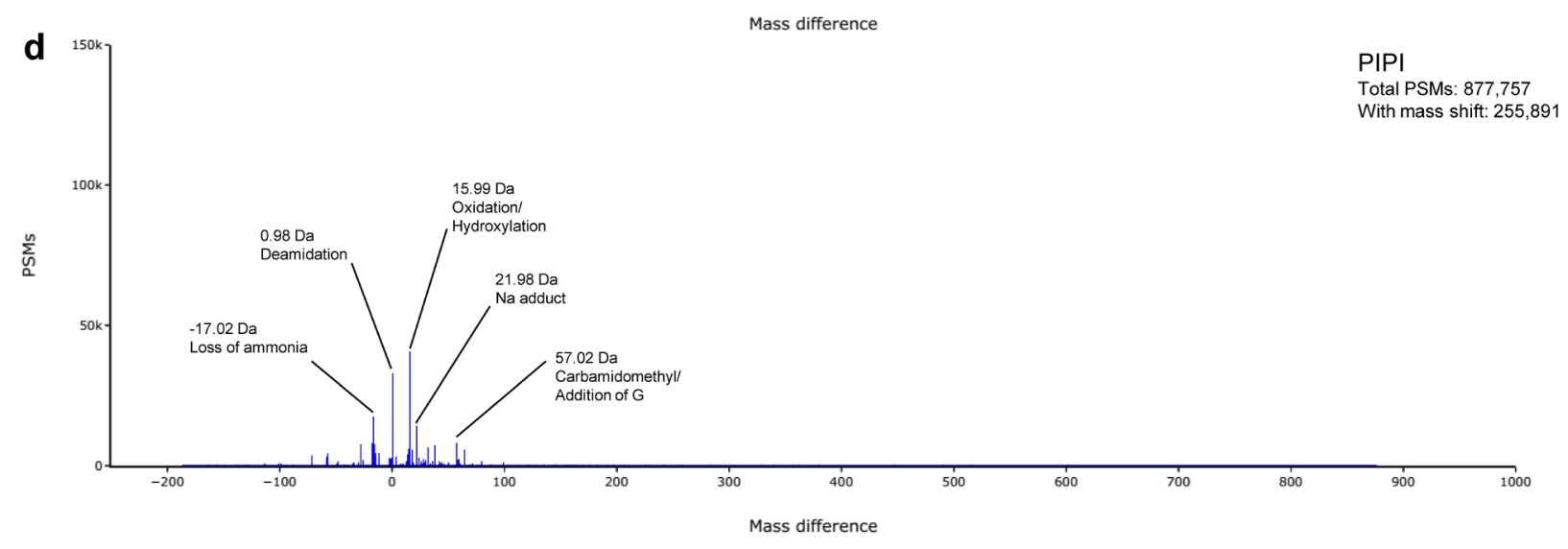

Figure S6. Mass difference profiles after unified post-processing of OMS results for $\boldsymbol{E}$. coli. Mass differences from combined results of all employed OMS engines (a), filtered by $1 \%$ combined PEP, as well 
as mass differences from results of MSFragger (b), MODa (c), and PIPI (d), filtered by 1\% individual PEP, were post-processed using PTM-Shepherd. Results are presented as histograms giving the number of PSMs for each 0.05 $\mathrm{Da}$ mass difference bin, omitting PSMs with no mass difference, or mass differences corresponding to isotopic peak selection. Results were sanitized on the individual engine level (b-c) or for the combination of all engines (a) before post-processing with PTM-Shepherd. Annotations for the most prominent mass differences are given. The number of all identified PSMs ("Total PSMs") is given for all subfigures as well as the number of PSMs that contain mass shifts shown in the profiles ("With mass shift"). It should be noted that PSMs containing multiple mass differences are counted multiple times. 


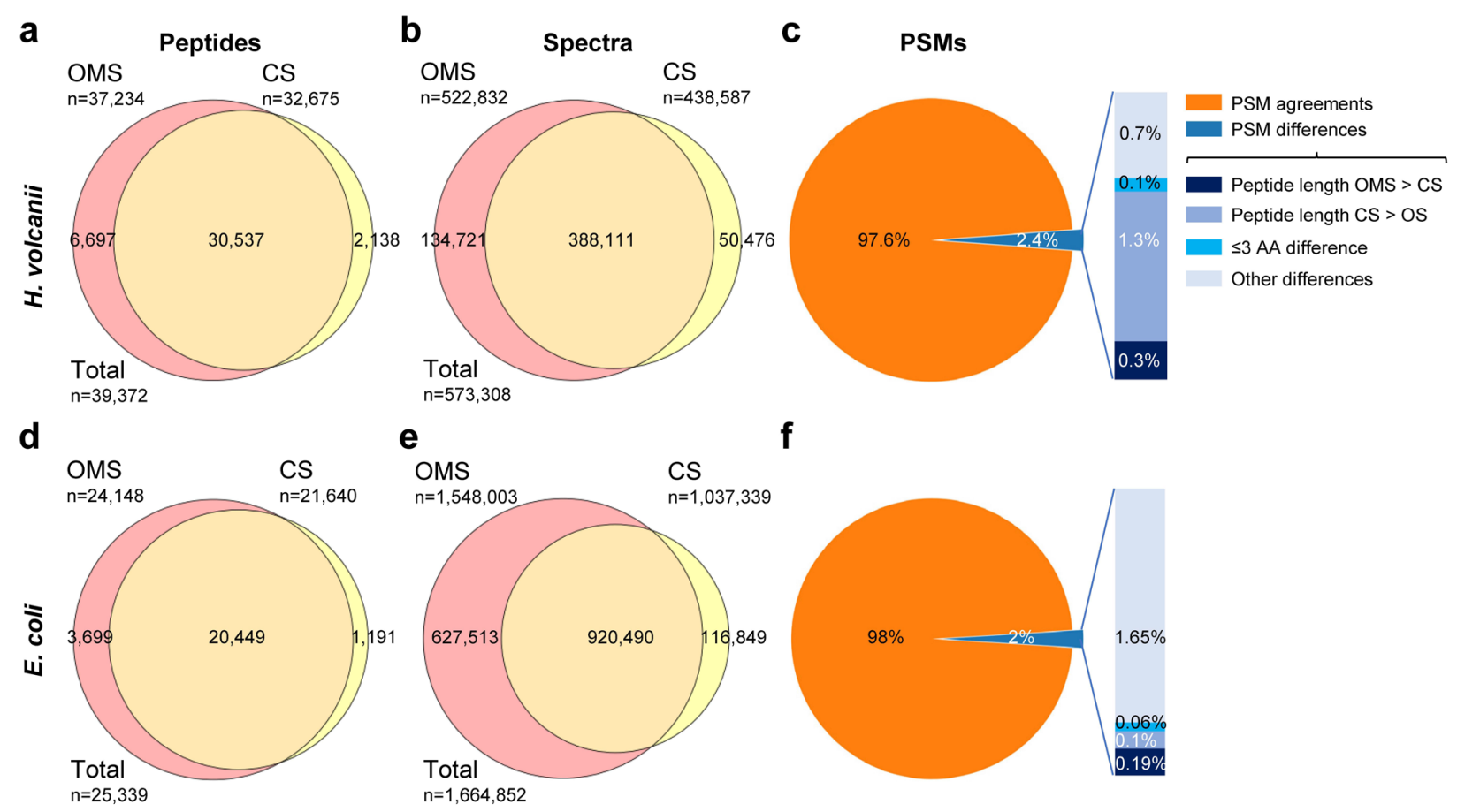

Figure S7. Comparisons between OMS and CS results showed similar trends for prokaryotic datasets as for the human dataset. Combined results from three OMS engines (red; MSFragger, MODa and PIPI) are compared to combined results from three CS engines (yellow; MSFragger, MS-GF+ and X!Tandem) for identified peptide sequences $(\mathbf{a}, \mathbf{d})$ and spectra $(\mathbf{b}, \mathbf{e})$. For commonly identified spectra, the percentage of PSM agreements (orange) and disagreements (blue) between the OMS and CS approach is given (c, f). Spectra with PSM disagreements are put in four categories: (i) spectra for which OMS engines matched a peptide sequences that is a substring of the one matched by CS engines (peptide length CS > OMS); (ii) spectra for which CS engines matched a peptide sequences that is a substring of the one matched by OMS engines (peptide length OMS $>$ CS); (iii) spectra for which the peptide sequences matched by OMS and CS engines differed by one to three amino acids; and (iv) spectra with peptide sequences differing between OMS and CS in any other way. Results are shown for the $H$. volcanii dataset $(\mathrm{a}, \mathrm{b}, \mathrm{c})$ as well as the E. coli dataset ( $\mathrm{d}, \mathrm{e}, \mathrm{f})$ and were filtered by $1 \%$ combined PEP and sanitized for each approach separately. 


\section{a}

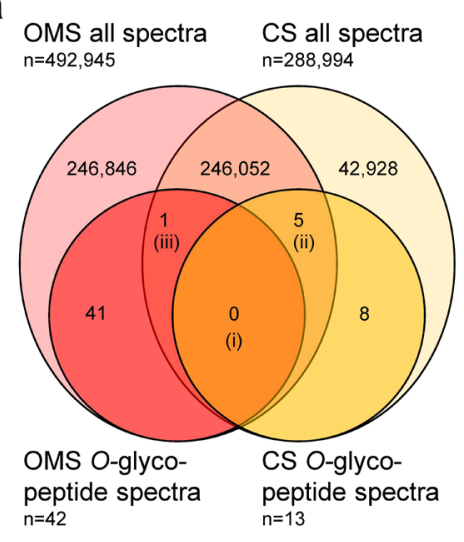

d

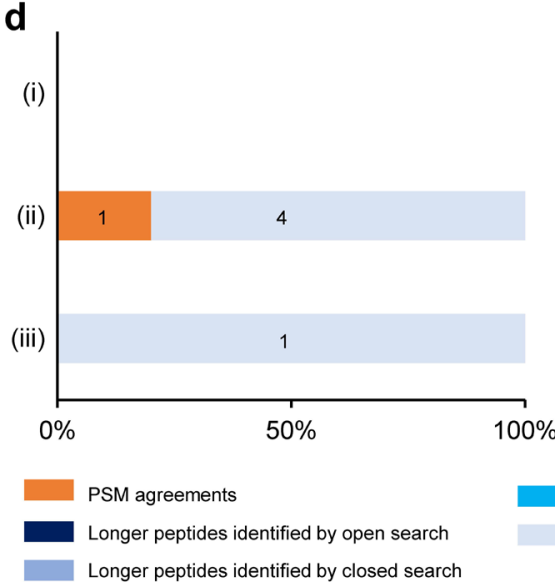

b

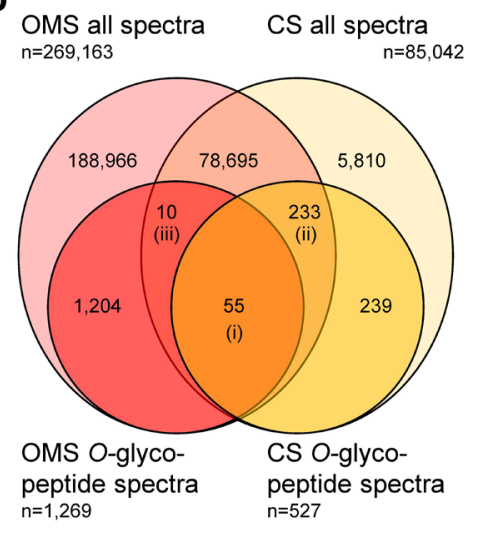

e

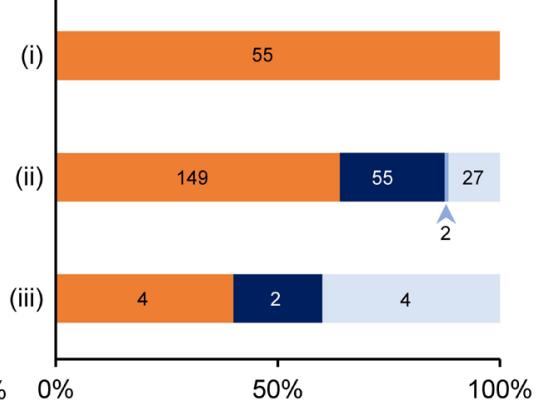

C

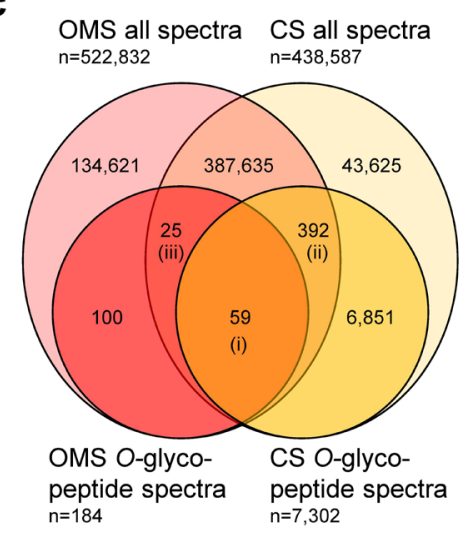

f

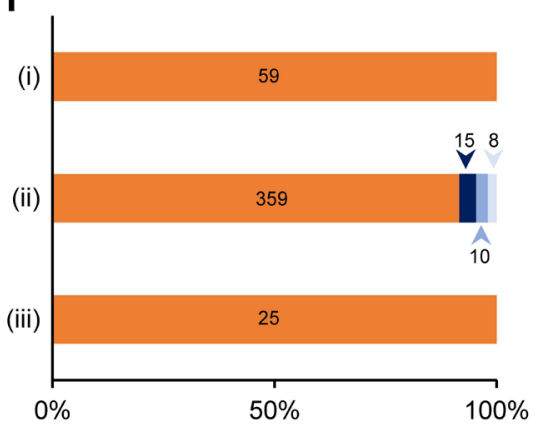

$\leq 3$ AA difference

Other differences

Figure S8. The identification of $\boldsymbol{O}$-glycopeptides remains challenging for OMS engines. Venn diagrams are shown comparing all spectra identified by the combined OMS approach (light red), with a subset of spectra matched to peptides containing mass shifts that were mapped to known $O$-glycans (dark red), and all spectra identified by the combined CS approach (light yellow), with a subset of spectra matched to $O$ glycopeptides (dark yellow). These comparisons are shown for (a), the H. sapiens in-depth proteomics dataset (PXD004452), (b) the H. sapiens glycoproteomic dataset (PXD013715), and (c), the H. volcanii dataset (PXD021874). Results were filtered by 1\% combined PEP and sanitized for each approach separately. For each subfigure, three groups of spectra are analyzed in more detail: (i) spectra with $O$ glycopeptides identified in both approaches, CS and OMS; (ii) spectra matched to $O$-glycopeptides by CS engines but for which OMS engines identified peptides with mass shifts that could not be mapped to known $O$-glycans; and (iii) spectra matched by OMS engines to peptides with mass shifts that are annotated as $O$ glycans while the CS approach did not match them to $O$-glycopeptides. (d), (e), and (f) present PSM agreements (orange) and differences (blue) in each of these three categories for the datasets corresponding to (a), (b), and (c), respectively. The following types of differences are considered: peptide sequences with differing lengths, for which either OMS or CS identified the shorter peptide that is a substrings of the longer one; peptides with one to three differing amino acids; and peptides differing in any other way (considered clear disagreements). It should be noted that results from CSs can include multiple glycans per peptide, while combinations of glycans have not been included in the matching of OMS results. 